\title{
Atomic-Scale Determination of Misfit Dislocation Loops at Metal-Metal Interfaces
}

Jacobsen, Joachim; Nielsen, L. Pleth; Besenbacher, Flemming; Stensgaard, Ivan; Lægsgaard, Erik; Rasmussen, Torben; Jacobsen, Karsten Wedel; Nørskov, Jens Kehlet

Published in:

Physical Review Letters

Link to article, DOI:

10.1103/PhysRevLett.75.489

Publication date:

1995

Document Version

Publisher's PDF, also known as Version of record

Link back to DTU Orbit

Citation (APA):

Jacobsen, J., Nielsen, L. P., Besenbacher, F., Stensgaard, I., Lægsgaard, E., Rasmussen, T., Jacobsen, K. W., \& Nørskov, J. K. (1995). Atomic-Scale Determination of Misfit Dislocation Loops at Metal-Metal Interfaces. Physical Review Letters, 75(3), 489-492. https://doi.org/10.1103/PhysRevLett.75.489

\section{General rights}

Copyright and moral rights for the publications made accessible in the public portal are retained by the authors and/or other copyright owners and it is a condition of accessing publications that users recognise and abide by the legal requirements associated with these rights.

- Users may download and print one copy of any publication from the public portal for the purpose of private study or research.

- You may not further distribute the material or use it for any profit-making activity or commercial gain

- You may freely distribute the URL identifying the publication in the public portal 


\title{
Atomic-Scale Determination of Misfit Dislocation Loops at Metal-Metal Interfaces
}

\author{
J. Jacobsen, ${ }^{1,2}$ L. Pleth Nielsen, ${ }^{1,3}$ F. Besenbacher, ${ }^{1,3}$ I. Stensgaard, ${ }^{1,3}$ E. Lægsgaard, ${ }^{1,3}$ \\ T. Rasmussen, ${ }^{1,2}$ K. W. Jacobsen, ${ }^{1,2}$ and J. K. Nørskov ${ }^{1,2}$ \\ ${ }^{1}$ Center for Atomic-Scale Materials Physics, Technical University of Denmark, DK 2800 Lyngby, Denmark \\ ${ }^{2}$ Physics Department, Technical University of Denmark, DK 2800 Lyngby, Denmark \\ ${ }^{3}$ Institute of Physics and Astronomy, University of Aarhus, DK 8000 Aarhus C, Denmark
}

(Received 26 January 1995)

\begin{abstract}
The growth of one monolayer of $\mathrm{Au}$ on $\mathrm{Ni}(111)$ is shown to lead to an ordered array of misfit dislocation loops in the underlying $\mathrm{Ni}(111)$ surface. The signature of these loops is observed by scanning tunneling microscopy, and atomistic simulations are used to relate the observed surface structure to that of the buried interface. The new interface structure is different from normal misfit dislocation structures in three respects: (i) it forms already during growth of a single Au monolayer, (ii) it forms in the substrate and not in the overlayer, and (iii) it is controlled by the interface energy rather than by the strain in the two phases.
\end{abstract}

PACS numbers: 68.35.Fx, 61.16.Ch, 61.72.Lk, 68.35.Md

The structure of the interphase between two different materials is determined by a detailed balance between the structure of the two bulk phases and the interatomic interactions at the interface. As early as 1949 it was suggested by Frank and van der Merwe [1] that the stress induced by the mismatch in the interface may be relieved through the formation of misfit dislocations, that is, regions with large changes in the interatomic distances separating domains with more normal bond lengths. These misfit dislocations affect not only the adhesive properties, and hence the strength of the interface, but also the electronic properties. A large number of electronmicroscopy studies of interface structures have described misfit dislocations on the micron scale [2], but the detailed atomic structure is much harder to obtain.

Recent scanning tunneling microscopy (STM) studies have observed the formation of misfit dislocations during the growth of one metal on another. For $\mathrm{Ag}$ on $\mathrm{Pt}(111)$ [3] and $\mathrm{Cu}$ on $\mathrm{Ru}(0001)$ [4], it is found that initially a pseudomorphically strained first layer is formed, followed by subsequent layers in which the strain is relieved either unidirectionally (1D domain walls) or more isotropically in two-dimensional (2D) dislocation networks in the overlayer. Similar observations have been published for EuTe on PbTe(111), although the thickness of the films was considerably larger in this case [5]. These systems nicely illustrate the concept of a critical layer thickness, beyond which the strain in a pseudomorphic overlayer is so large that it must be relieved through the formation of misfit dislocations [2].

In this Letter, we present a new scenario for the matching between two metals with large lattice mismatch $(16 \%)$. We show that the growth of a single monolayer of $\mathrm{Au}$ on $\mathrm{Ni}(111)$ results in an ordered array of triangular misfit dislocation loops in the underlying Ni surface. The signature of these loops is revealed in STM images of the surface structure, and atomistic simulations within the effective-medium theory relate this to the atomic structure at the buried interface. For this system, the misfit dislocations are formed in the substrate and not in the overlayer as previously observed for other systems. We furthermore conclude that the origin of the novel misfit dislocation structure is closely related to the large lattice misfit and the strong $\mathrm{Au}-\mathrm{Ni}$ interactions at the interface.

The experiments were performed with a high-stability STM operating in ultrahigh vacuum [6]. The Au evaporations $(0.1 \mathrm{ML} / \mathrm{min})$ were performed by resistively heating a small $\mathrm{Au}$ droplet.

If we deposit $\mathrm{Au}$ on a cold $(T=170 \mathrm{~K}) \mathrm{Ni}(111)$ surface and allow it to anneal slowly to room temperature (RT), an incommensurable Au moiré overlayer structure [Fig. 1(a)] results. The corrugation of the moire structure is $0.5 \AA$, and those regions which are imaged low are those at which the $\mathrm{Au}$ atoms are close to on-top positions with respect to the underlying $\mathrm{Ni}$ lattice, opposed to what one would intuitively expect from a simple hardball model. This is a consequence of substrate relaxations around on-top adsorbate positions [7]. The periodicity of the moiré pattern is close to $9 \times 9(9.1 \pm 0.1)$ with respect to the underlying $\mathrm{Ni}$ lattice. The ratio of the first-layer $\mathrm{Au}$ - $\mathrm{Au}$ distance $(2.80 \pm 0.10 \AA)$ to the $\mathrm{Ni}-\mathrm{Ni}$ distance $(2.49 \AA)$ in the underlying surface layer accounts for the $9 \times 9$ periodicity.

If, on the other hand, we deposit Au at RT or anneal the low-temperature structure to $\sim 400 \mathrm{~K}$, the structures seen in Figs. 1(b)-1(d) result. The high mobility of the deposited Au atoms at RT or above causes the $\mathrm{Au}$ atoms to flow to ascending step edges where the $\mathrm{Au}$ nucleates and grows in large dendritic islands with a local $\mathrm{Au}$-surface coverage corresponding to a full monolayer [Fig. 1(b)]. As depicted, the monatomic high (2.5 $\AA) \mathrm{Au}$ islands are easily discernible from $\mathrm{Ni}$ at the step edges ( $0.4 \AA$ higher). It is found that the Au covered areas 

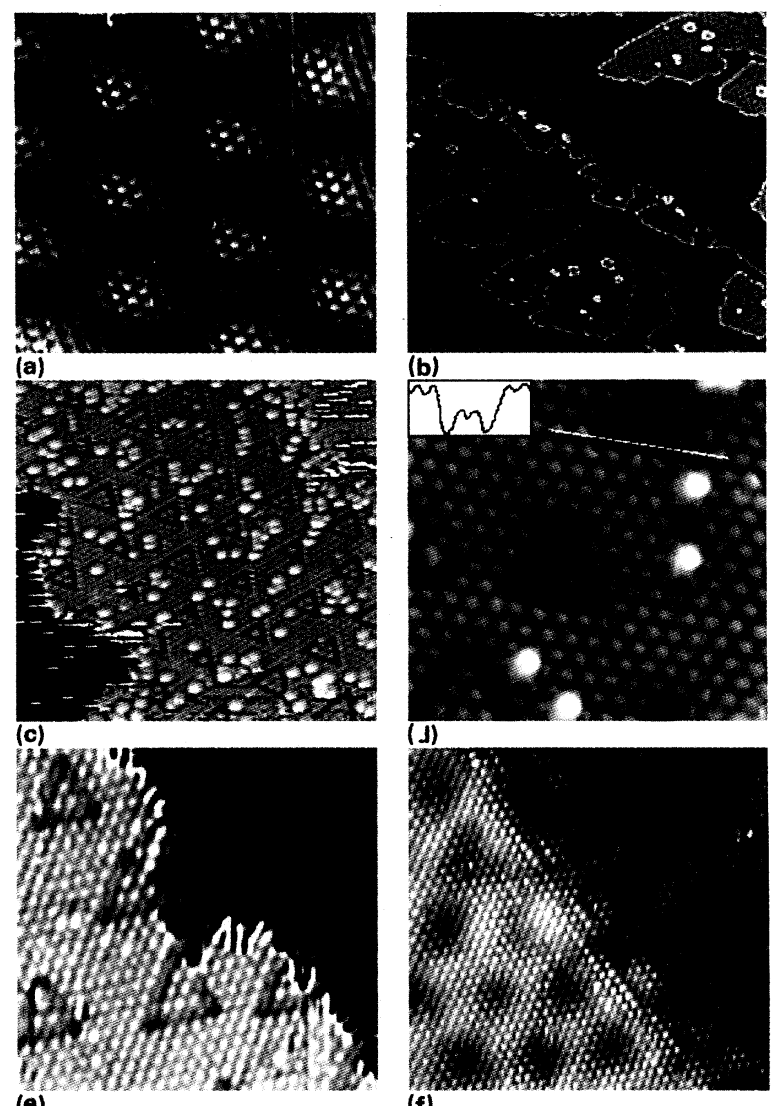

(e)

(f)

FIG. 1. STM topographs showing (a) $\left(70 \times 75 \AA^{2}\right)$ a $9 \times 9$ moiré overlayer structure after depositing $\mathrm{Au}$ at $170 \mathrm{~K}$ (the $\mathrm{Au}$ and the moiré lattices are rotated $2^{\circ}$ and $13^{\circ}$, respectively, relative to the Ni lattice); (b) $\left(1450 \times 1570 \AA^{2}\right) \mathrm{Au}$ islands at $\mathrm{Ni}$ step edges; (c) $\left(145 \times 160 \AA^{2}\right)$ revealing the $9.7 \times$ 9.7 triangular structure in a $\mathrm{Au}$ island growing out from a descending $\mathrm{Ni}$ step edge (upper right); (d) $\left(45 \times 50 \AA^{2}\right)$ the atomic details of the triangular superstructure; (e) $\left(70 \times 75 \AA^{2}\right)$ a coexisting area of the $\mathrm{Ni}(111)$ substrate and the Au structure; (f) $\left(95 \times 104 \AA^{2}\right)$ second and third Au layers separated by an underlying monatomic $\mathrm{Ni}$ step. The bright regions on the $\mathrm{Au}$ areas in (b) are associated with the initial growth of second layer $\mathrm{Au}$ islands.

on separate terraces are directly proportional to the area of the terraces on which the Au impinges, indicating the existence of a significant barrier for diffusing across step edges.

Zooming in on the first-layer Au islands [Figs. 1(c) and 1(d)], a periodic network of triangles is revealed. The average distance between the center of neighboring triangles is $9.7 \pm 0.2$ times the interatomic distance on the bare Ni(111) surface. The equilibrium distance between the individual $\mathrm{Au}$ atoms in the first layer [Fig. 1(d)] is still $2.80 \pm 0.10 \AA$, that is, slightly less than the Au-Au distance $(2.88 \AA)$ of the nonreconstructed $\mathrm{Au}(111)$ surface, but very close to the $\mathrm{Au}-\mathrm{Au}$ distance in the herringbone reconstruction of the $\mathrm{Au}(111)$ surface, for which the Au- surface layer density is increased by $4.4 \%$ [8]. From STM images of areas [Fig. 1(e)] in which the superstructure coexists with the bare $\mathrm{Ni}(111)-(1 \times 1)$ surface, it can be concluded that neither the trigonal superstructure nor the $\mathrm{Au}$ lattice in the superstructure are rotated relative to the underlying substrate.

Figures 1(c)-1(e) show that the triangular structures surround small Au clusters of variable size $(0,1,3$, 6,10 , or $15 \mathrm{Au}$ atoms), depending on the length of the surrounding triangular lines. The registry of the $\mathrm{Au}$ layer with respect to the underlying $\mathrm{Ni}$ layer is such that the triangular structures appear close to ontop positions relative to the underlying Ni lattice. The depth of the triangular structures or lines is only $0.3-$ $0.5 \AA$, indicating that the triangular structures cannot be attributed to vacancies in the Au layer. It is also evident that faint protrusions exist in the triangular lines which are in registry with the surrounding Au lattice. We also note that distinct protrusions $(\sim 0.2 \AA$ high $)$ appear to be substituted into the flat portions of the Au surface.

The origin of this triangular network in the Au overlayer is revealed by atomistic simulations within the effective-medium theory. This approximate method has been shown previously to give a very reasonable description of fcc metals in general [9] and the $\mathrm{Au} / \mathrm{Ni}$ system in particular [10]. The equilibrium structures have been determined using a simulated annealing technique. The dynamics of the system is described using molecular dynamics coupled to a heat bath, the temperature of which is increased to $700 \mathrm{~K}$ and subsequently cooled down to RT over 100 ps.

We have found two very different stable configurations of the system with a monolayer of $\mathrm{Au}$ on $\mathrm{Ni}(111)$, and we have studied both states as a function of the periodicity of the superstructure formed. The first state is obtained by placing an $(N-1) \times(N-1)$ Au layer on top of an $N \times N \mathrm{Ni}(111)$ substrate and calculating the equilibrium configuration by minimizing the energy with respect to the coordinates of all atoms in the system. Figure 2(a) shows the energy as a function of $N$. It is found that the equilibrium superstructure [Fig. 3(a)] corresponds approximately to the experimentally observed $9 \times 9$ moiré overlayer structure [Fig. 1(a)].

If, on the other hand, inspired by the experimental observations, we remove a row of five $\mathrm{Ni}$ atoms from the first $\mathrm{Ni}$ layer and incorporate these in the Au layer, a new equilibrium configuration results. The equilibrium structure [Fig. 3(b)] in the Au overlayer is very similar to the triangular structure observed in the STM topographs [Figs. 1(b)-1(e)]. From Fig. 2(a) it is seen that in this case the minimum-energy superstructure periodicity $(N)$ is larger, close to 10 , as compared to the simple moiré superstructure, and in good agreement with the $9.7 \times 9.7$ periodicity observed by STM. The origin of the new configuration is revealed from the buried $\mathrm{Ni}$ layer [Fig. 3(c)]. Here the formation of the Ni vacancies 


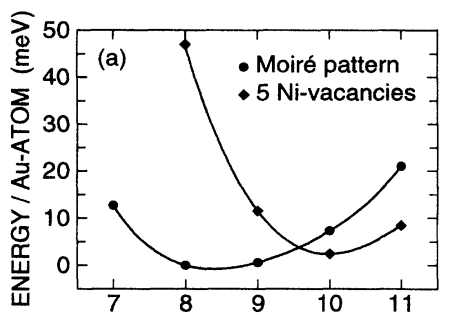

SUPERSTRUCTURE PERIODICITY

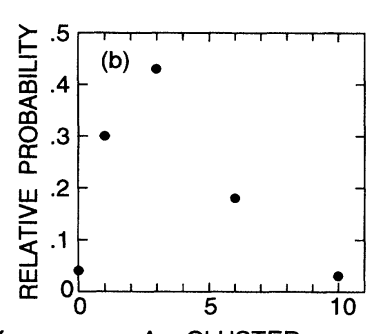

AU CLUSTER
FIG. 2. (a) Calculated energy per Au atom for two different surfaces structures as a function of the superstructure periodicity: A simple moiré overlayer, and a structure formed by substituting five $\mathrm{Ni}$ atoms from the topmost $\mathrm{Ni}$ layer into the $\mathrm{Au}$ overlayer. (b) The experimentally obtained distribution of triangular misfit dislocations vs the center Au cluster size obtained by counting 380 triangles.

have induced a shift of the $\mathrm{Ni}$ atoms within a triangular area from being in fcc sites to hcp sites [11]. The triangular boundary separating the fcc and the faulted hcp regions is clearly a partial dislocation loop, and it is the signature of this buried interface vacancy misfit dislocation that is revealed as the triangular structure in the STM topographs. From the STM topographs, it is found that the triangular corner atoms are positioned lower than the $\mathrm{Au}$ atoms along the loop [line scan, Fig. 1(d)], a fact that is confirmed by the calculations.

The energy of the triangular structure is found to be extremely close (within $2 \mathrm{meV}$ per Au atom) to the lowest energy state of the moiré overlayer structure. However, it should be emphasized that such a small energy difference is certainly within the uncertainty of an approximate totalenergy method like the effective-medium theory. In fact, the energy difference is so small that the free-energy difference at RT could easily have the other sign due to the entropy associated with the disordered $\mathrm{Ni}$ in the $\mathrm{Au}$ overlayer (the entropy associated with distributing

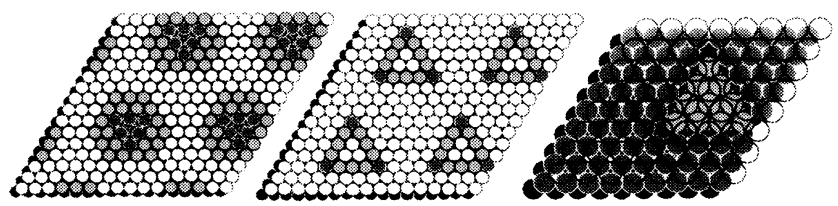

(a)

(b)

(c)

FIG. 3. Equilibrium structures from the simulations of an $8 \times 8 \mathrm{Au}$ overlayer on a $9 \times 9 \mathrm{Ni}(111)$ surface. (a) Moiré overlayer structure where the low lying areas correspond to substrate on-top positions, and (b) the surface structure when five $\mathrm{Ni}$ atoms are moved from the topmost $\mathrm{Ni}$ layer. The shading in (a) and (b) indicates the height of the individual atoms with dark representing the lowest lying atoms. (c) The structure of the underlying Ni layer revealing a triangular misfit dislocation loop. The Au overlayer atoms are shown by open circles. The dark grey Ni atoms occupy fcc positions, whereas the light grey $\mathrm{Ni}$ atoms are shifted to hcp sites, thereby allowing the $\mathrm{Au}$ overlayer atoms to coordinate to three $\mathrm{Ni}$ atoms as opposed to one.
$5 \mathrm{Ni}$ atoms over 64 sites gives an entropy contribution to the free energy of $-6 \mathrm{meV}$ per Au atom at RT). The main result from the simulations is, however, the finding of two distinct structures that closely resemble the two experimentally observed structures.

It is interesting to investigate why the triangular structure has an energy in the same range as the moiré structure, in spite of the large and positive energy involved in forming the partial dislocation loops and the hcp regions. The main reason is that the fcc-to-hcp shift of the $\mathrm{Ni}$ atoms in the triangular area makes it possible for the cluster of $\mathrm{Au}$ atoms in the overlayer to coordinate to three $\mathrm{Ni}$ atoms underneath rather than to one. The $\mathrm{Ni}$ atoms, which are squeezed out from the topmost Ni layer during the formation of the partial dislocation loops, are alloyed into the $\mathrm{Au}$ overlayer. Experimentally these $\mathrm{Ni}$ atoms are revealed from the STM topographs as randomly distributed distinct protrusions, preferentially alloyed into the Au layer outside the triangular loops. The fact that the $\mathrm{Ni}$ atoms for most tip configurations are imaged higher than the surrounding $\mathrm{Au}$ atoms has also been observed previously for the $\mathrm{Au}-\mathrm{Ni}(110)$ system [10]; this behavior must be ascribed to electronic effects. As a further confirmation of the model it is found [Fig. 1(c)] that there is one-to-one correspondence between the amount of $\mathrm{Ni}$ squeezed out of the Ni-surface layer to form the dislocation loops and the "white Ni protrusions" alloyed into the Au surface layer. It may seem strange that this happens since $\mathrm{Au}$ and $\mathrm{Ni}$ are immiscible in the bulk, but it is known from previous experimental and theoretical work that Au-Ni surface alloys do form [10].

The scenario for the formation of the Au-Ni(111) interface is thus very different from the previously studied systems $[3,4]$. First, the mismatch is so large and the Au$\mathrm{Au}$ interactions so strong that a pseudomorphic overlayer is never formed. The low-temperature moiré structure already shows a $\mathrm{Au}$ overlayer with approximately the equilibrium Au-Au distance. However, this structure is not particularly stable because some of the Au atoms are placed in on-top or close to on-top positions with respect to the underlying $\mathrm{Ni}$ atoms. The driving force for the formation of the misfit dislocations is therefore not the strain induced by forcing the overlayer to have the lattice constant of the substrate but rather the $\mathrm{Au}-\mathrm{Ni}$ interactions in the parts of the interface where the bonding is unfavorable. The reason that the vacancy-dislocation loops are formed only at RT or above is simply that the activation energy involved to break the $\mathrm{Ni}-\mathrm{Ni}$ bonds has to be overcome.

In the model discussed above, the different sizes of the observed triangular structures depend on the number of removed $\mathrm{Ni}$ atoms and hence reflect the sizes of the hcp regions. Figure 2(b) shows the experimentally found distribution of misfit dislocation triangles as a function of the center Au-cluster size or, equivalently, the number of associated $\mathrm{Ni}$ vacancies. It is found that at RT there 
is a predominance of triangles with $\mathrm{Au}$ trimers. The reason for this optimum may be as follows: We have argued above that the driving force for the formation of the triangles is simply that $\mathrm{Au}$ overlayer atoms do not want to be in Ni on-top positions. There are, however, only a few Au overlayer atoms per unit cell of the moiré lattice that are directly on top with respect to the Ni lattice. This means that as the triangle becomes larger, the driving force becomes weaker, and since there are energy costs associated with the formation of the misfit dislocations in the $\mathrm{Ni}$ layer, there must be an optimum size of the triangles with quite a few atoms involved. It should be stressed that entropy effects could also play a role here.

The analysis above has addressed the interface structure for a monolayer-thick Au film on Ni(111). The question is what happens as more Au layers are deposited. From STM topographs at various Au layer thicknesses, it is found that reminiscences of the triangular misfit dislocations are still visible in the sequential Au layers [Fig. 1(f)]. However, the overall corrugation amplitude of the surface structure decreases significantly with the number of deposited Au layers, as shown in Fig. 4, which also includes the result of a simulation where more $\mathrm{Au}$ layers are added to the structure of Fig. 2(b). It is found that already the second layer is a nearly uniform hexagonal $\mathrm{Au}$ layer without any $\mathrm{Ni}$ atoms alloyed into it. We can thus conclude that the triangular misfit dislocation loops and the squeezed-out $\mathrm{Ni}$ remain at the interface, and that

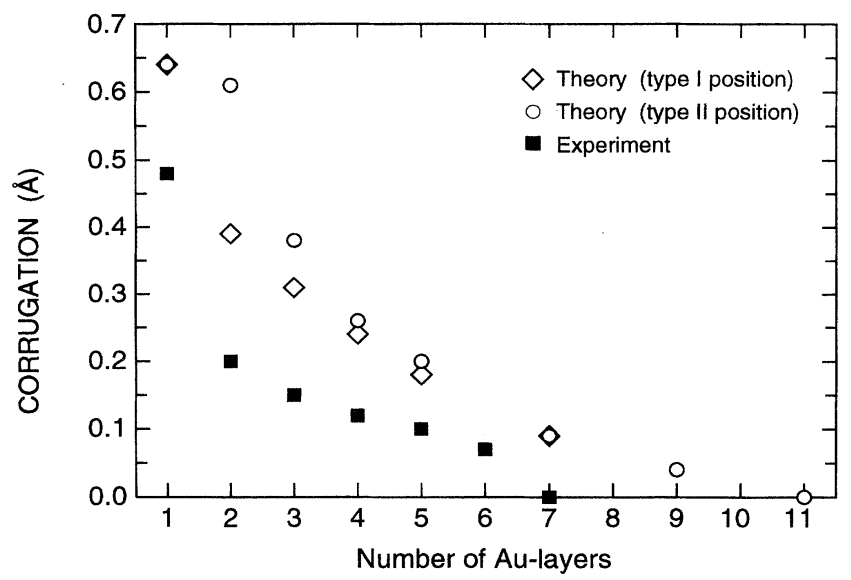

FIG. 4. The corrugation of the superstructure obtained from (i) STM topographs as a function of overlayer thickness and (ii) simulations for two Au overlayers with different stackings. the signature in the uppermost Au layer simply decreases with increasing thickness.

The results presented in this Letter represent an atomby-atom identification of the buried interface structure between two metals with a very large lattice mismatch. We have shown that an interplay between STM experiments and atomistic simulations provides a very powerful tool for revealing the structure of buried interfaces from the signatures at the topmost layer. We have also shown that the driving force in the formation of misfit dislocations in this system is controlled by the strong interface energy rather than the strain in the two phases.

CAMP, the Center for Atomic-Scale Materials Physics, is funded by the Danish National Research Foundation. Further funding is obtained from the Danish Research Councils through the Center for Nano-Tribology. Discussions with R. M. J. Cotterill are gratefully acknowledged.

[1] F. C. Frank and J. H. van der Merwe, Proc. R. Soc. London 198, 205 (1949)

[2] For a review, see, e.g., J.W. Mattheus, in Epitaxial Growth (Academic Press, New York, 1975), Pt. B, p. 559.

[3] H. Brune, H. Röder, C. Boragno, and K. Kern, Phys. Rev. B 49, 2997 (1994).

[4] G. O. Pötschke and R. J. Behm, Phys. Rev. B 44, 1442 (1991).

[5] N. Frank, G. Springholz, and G. Bauer, Phys. Rev. Lett. 73, 2236 (1994).

[6] L. Eierdal, F. Besenbacher, E. Lægsgaard, and I. Stensgaard, Surf. Sci. 312, 31 (1994). The microscope is the prototype of Rasterscope 3000 from DME, Herlev, Denmark.

[7] S. M. Foiles, Surf. Sci. 292, 5 (1993).

[8] U. Harten, A. M. Lahee, J.P. Toennies, and Ch. Wöll, Phys. Rev. Lett. 54, 2619 (1985); J. V. Barth, H. Brune, G. Ertl, and R. J. Behm, Phys. Rev. B 42, 9307 (1990).

[9] The details of the theoretical method as well as the parameters used can be found in K. W. Jacobsen, J. K. Nørskov, and M. J. Puska, Phys. Rev. B 35, 7423 (1987); P. Stoltze, J. Phys. Condens. Matter 6, 9495 (1994).

[10] L. Pleth Nielsen, F. Besenbacher, I. Stensgaard, E. Lægsgaard, C. Engdahl, P. Stoltze, K. W. Jacobsen, and J. K. Nørskov, Phys. Rev. Lett. 71, 754 (1993); 74, 1159 (1995).

[11] It has earlier been speculated that such structures should exist; see R. M. J. Cotterill, in Lattice Defects in Quenched Metals (Academic Press, New York, 1965), p. 97; F. W. Schapink and M. De Jong, Acta Metall. 12, 755 (1964). 

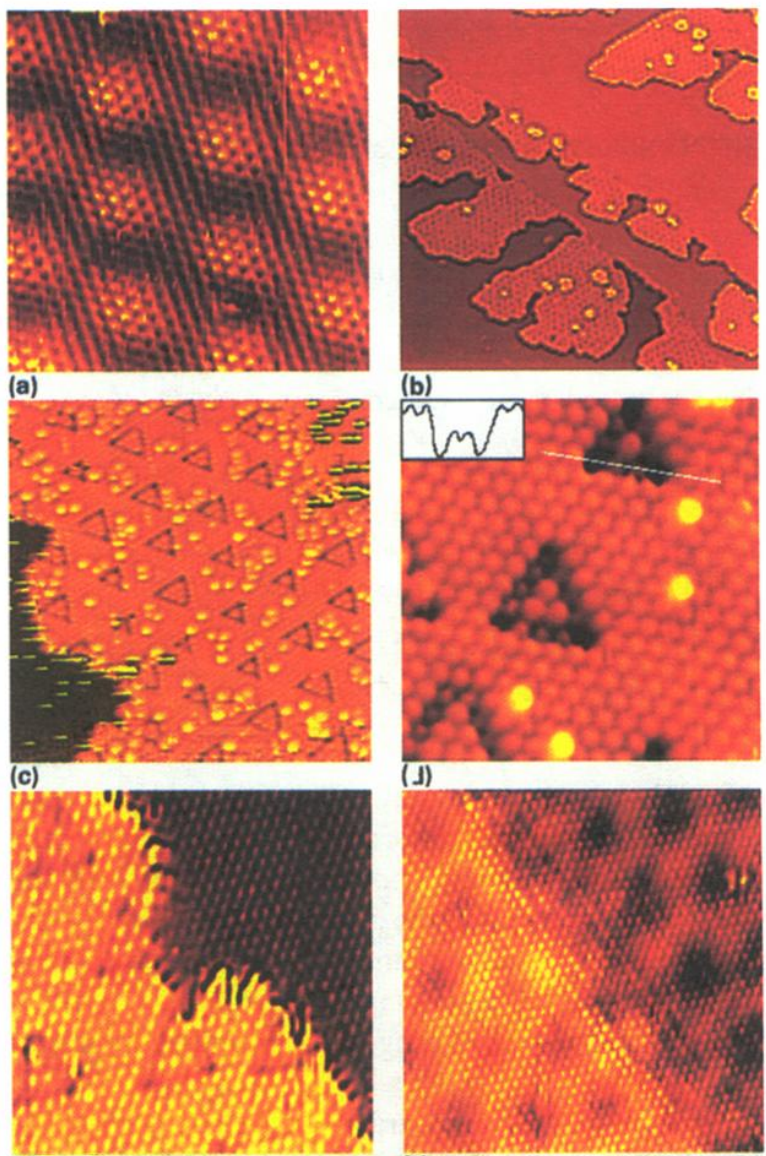

(e)

(f)

FIG. 1. STM topographs showing (a) $\left(70 \times 75 \AA^{2}\right)$ a $9 \times 9$ moiré overlayer structure after depositing Au at $170 \mathrm{~K}$ (the $\mathrm{Au}$ and the moiré lattices are rotated $2^{\circ}$ and $13^{\circ}$, respectively, relative to the $\mathrm{Ni}$ lattice); (b) $\left(1450 \times 1570 \AA^{2}\right) \mathrm{Au}$ islands at $\mathrm{Ni}$ step edges; (c) $\left(145 \times 160 \AA^{2}\right)$ revealing the $9.7 \times$ 9.7 triangular structure in a $\mathrm{Au}$ island growing out from a descending Ni step edge (upper right); (d) $\left(45 \times 50 \AA^{2}\right)$ the atomic details of the triangular superstructure; (e) $\left(70 \times 75 \AA^{2}\right)$ a coexisting area of the $\mathrm{Ni}(111)$ substrate and the Au structure; (f) $\left(95 \times 104 \AA^{2}\right)$ second and third Au layers separated by an underlying monatomic $\mathrm{Ni}$ step. The bright regions on the Au areas in (b) are associated with the initial growth of second layer $\mathrm{Au}$ islands. 